\title{
L'efficacia, la sicurezza e la maneggevolezza del sodio oxibato: i risultati dello studio GUM (GHB-Use and Misuse)
}

\author{
Ezio Manzato*, Felice Nava*, Gianmario Borroni**, Elena Bosticco**, Silvia Bravin**, \\ Giuseppe Fertonani Affini**, Gianluigi Gasparini**, Maura Richini**, Mauro Thoux**, \\ Marta Torriani**, Alfio Lucchini**
}

\section{Introduzione}

L'agonista dell'acido gamma-aminobutirrico (GABA) sodio oxibato $(50-100 \mathrm{mg} / \mathrm{kg} / \mathrm{die})$ è prescritto per il trattamento dell'alcolismo in quanto si è dimostrato efficace nel sopprimere $\mathrm{i}$ sintomi della sindrome di astinenza da alcol (AWS) e nel mantenere l'astinenza, controllando il craving alcolico e prevenendo le ricadute nel bere pesante, usato in tre o sei somministrazioni (nel caso di alcolisti "non responder" all'usuale somministrazione di tre dosi). Il farmaco è stato approvato per il trattamento della dipendenza da alcol dagli organismi regolatori in Italia e in Austria (Addolorato e coll., 1996, 1998, 1999a, b, 2000; Caputo e coll., 2009a, 2013; Nava e coll., 2006, 2007, 2010; Keating, 2014; Skala e coll., 2014). Tuttavia il sodio oxibato ha mostrato di avere proprietà additive e pertanto è necessario tenere presente il suo rischio potenziale di abuso in particolare in alcuni sottogruppi di pazienti con dipendenza da alcol e associata comorbilità psichiatrica, specialmente il disturbo borderline di personalità (BPD), o dipendenza attuale o in remissione completa da cocaina ed eroina.

In tali tipologie di alcolisti il farmaco non dovrebbe essere prescritto, mentre può essere utilizzato in modo sicuro negli alcolisti "puri" (Addolorato e coll., 2000; Caputo e coll., 2009a, b, 2011; Keating, 2014; Skala e coll., 2014). In particolare, per quanto riguarda il potenziale di craving, di abuso e di sintomi astinenziali correlati all'uso del sodio oxibato nel trattamento della dipendenza da alcol, il rischio di abuso del sodio oxibato è generalmente basso $(\approx 10 \%$ dei soggetti trattati) quando il farmaco è somministrato ad alcolisti al dosaggio raccomandato (50-100 $\mathrm{mg} / \mathrm{kg} / \mathrm{die}$ ), sotto supervisione di un membro della famiglia e con stretta sorveglianza medica continua (Caputo e coll., 2009a, b, 2015; Keating, 2014; Skala e coll., 2014).

\section{Caratteristiche dello studio}

Lo studio denominato con l'acronimo GUM (GHB-Use and Misuse) ha avuto carattere di inchiesta (survey) longitudinale di tipo retrospettivo.

* Dirigenti medici, FeDerSerD Nazionale.

** Dirigenti Medici, Centri Alcologici di Aosta, Ivrea (Torino), Abbiategrasso (Milano), Gorgonzola (Milano), Vallecamonica (Brescia), Zevio (Verona) e Parma.
L'osservazione è stata riferita a soggetti dipendenti da alcol in trattamento con sodio oxibato (Alcover ${ }^{\circledR}$ ) all'interno dei normali percorsi diagnostico-terapeutici dei singoli servizi di alcologia partecipanti allo studio.

Il periodo di osservazione è stato relativo agli anni 2005 , 2006, 2007.

\section{Centri Partecipanti allo Studio}

Sono stati selezionati in maniera randomizzata 10 centri clinici di alcologia del Nord Italia che hanno accettato di partecipare allo studio.

Di questi 7 hanno completato lo studio e sono stati i centri di Aosta, Ivrea (Torino), Abbiategrasso (Milano), Gorgonzola (Milano), Vallecamonica (Brescia), Zevio (Verona) e Parma.

\section{Breve descrizione dello studio}

\section{Criteri di inclusione ed esclusione}

I criteri di inclusione sono stati:

1. soggetti dipendenti da alcol secondo i criteri del DSMIV-TR;

2. soggetti in trattamento con sodio oxibato (Alcover ${ }^{\circledR}$ ) negli anni 2005, 2006 e 2007;

3. età 18-65.

I criteri di esclusione sono stati:

1. gravi patologie internistiche (es. diabete, cirrosi, ecc.);

2. gravi patologie neurologiche (es. epilessia, morbo di Parkinson, demenza, ecc.);

3. gravidanza.

\section{Obiettivi dello studio}

Gli obiettivi primari dello studio sono stati di valutare:

1. l'attività antiastinenziale;

2. il mantenimento dell'astinenza (prevenzione delle ricadute);

3. il drop-out dal trattamento.

Gli obiettivi secondari dello studio sono stati di valutare:

1. gli effetti collaterali e le reazioni avverse (ADR); 
2. i comportamenti di misuso (comportamenti "appetitivi");

3. gli episodi di intossicazione e di overdose (abuso).

\section{Strumenti per la valutazione}

Per la natura retrospettiva dello studio la valutazione degli end-points si è basata sulla raccolta di dati clinici contenuti nelle cartelle cliniche.

I dati clinici sono stati raccolti mediante l'utilizzo di due serie di schede (protocollo dello studio).

La prima serie di schede (costituita da un numero di sei) ha permesso di raccogliere per singolo paziente i dati su:

1. storia demografica (scheda n. 1) e tossicologica (scheda n. 2);
2. esami di laboratorio ematochimici e tossicologici (scheda n. 3);

3. esiti di questionari e test psicologici (scheda n. 4);

4. valutazione psicopatologica secondo i criteri del DSM-IV-TR (scheda n. 5);

5. definizione del programma terapeutico concordato (scheda n. 6).

La seconda serie di schede (costituita da un numero di sei) ha permesso di valutare a 6 e 12 mesi dall'inizio del trattamento i dati su:

1. andamento del programma terapeutico (scheda n. 1);

2. esito del trattamento farmacologico (scheda n. 2);

3. misuso (comportamenti "appetitivi"), abuso (intossicazioni e overdose) e astinenza (scheda n. 3);

Tab. 1 - Caratteristiche cliniche dei soggetti inclusi nello studio

\begin{tabular}{|c|c|c|}
\hline & Trattamento $<90$ giorni & Trattamento $>90$ giorni \\
\hline \multicolumn{3}{|l|}{ Caratteristiche demografiche } \\
\hline Età, anni (media + S.D.) & $45.68+1$ & $48.50+11.31$ \\
\hline Uomini $(\%)$ & 83 & 89 \\
\hline Coniugati $(\%)$ & 53 & 62 \\
\hline \multicolumn{3}{|l|}{ Educazione (\%): } \\
\hline Scuola Superiore & 26 & 32 \\
\hline Laurea & 5 & 6 \\
\hline \multicolumn{3}{|l|}{ Occupazione $(\%)$ : } \\
\hline Stabile occupazione & 69 & 71 \\
\hline Non stabile occupazione & 16 & 13 \\
\hline Disoccupato & 15 & 16 \\
\hline \multicolumn{3}{|l|}{ Situazione abitativa $(\%)$ : } \\
\hline Con la famiglia o amici & 68 & 63 \\
\hline Soli & 16 & 19 \\
\hline Con non fissa dimora & 14 & 16 \\
\hline In comunità terapeutica & 2 & 2 \\
\hline \multicolumn{3}{|l|}{ Abuso di alcol } \\
\hline Uso di alcol, anni (media + S.D.) & $12+9.03$ & $15+8.76$ \\
\hline Intake di alcol (media + S.D.) & $7.67+1.15$ & $8.72+1.22$ \\
\hline Alcol craving (media + S.D.) & $8.23+0.63$ & $8.08+0.89$ \\
\hline \multicolumn{3}{|l|}{ Policonsumatori (ultimi 12 mesi) (\%) } \\
\hline Policonsumatori (tutte le sostanze) & 37 & 39 \\
\hline Oppioidi & 18 & 18 \\
\hline Stimolanti & 5 & 7 \\
\hline Sedativi & 4 & 5 \\
\hline Sonniferi & 4 & 2 \\
\hline Più di una sostanza & 10 & 6 \\
\hline Giocatori patologici o altri dipendenti comportamentali & 1 & 0 \\
\hline Policonsumatori (in passato) (\%) & 21 & 28 \\
\hline Consumatori di tabacco (\%) & 84 & 76 \\
\hline Co-morbilità psichiatrica $(\%)$ & 36 & 39 \\
\hline Disturbi di Asse I & 25 & 21 \\
\hline Disturbi d'ansia & 7 & 11 \\
\hline Schizofrenia e disturbi psicotici & 4 & 4 \\
\hline Depressione e disturbi dell'umore & 14 & 16 \\
\hline Disturbi di Asse II & 12 & 9 \\
\hline Disturbi di personalità cluster $\mathrm{A}$ & 3 & 3 \\
\hline Disturbi di personalità cluster B & 7 & 6 \\
\hline Disturbi di personalità cluster $\mathrm{C}$ & 2 & 0 \\
\hline
\end{tabular}


4. esami di laboratorio ematochimici e tossicologici (scheda n. 4);

5. esiti di questionari e test psicologici (scheda n. 5);

6. evoluzione dello stato psicopatologico secondo i criteri del DSM-IV-TR (scheda n. 6).

\section{Caratteristiche cliniche dei soggetti inclusi nello studio}

Le caratteristiche cliniche dei soggetti inclusi nello studio sono rappresentate in Tab. 1.

In base all'esperienza clinica di utilizzo del sodio oxibato $\left(\right.$ Alcolver $\left.{ }^{\circledR}\right)$ i soggetti inclusi nello studio sono stati divisi in due gruppi: coloro che hanno ricevuto un trattamento per un periodo inferiore ai 90 giorni e coloro che lo hanno ricevuto per un periodo maggiore di 90 giorni.

Per i primi il trattamento è stato verosimilmente finalizzato al controllo della sindrome anti-astinenziale mentre per i secondi al mantenimento dell'astinenza. Entrambi i gruppi hanno presentato caratteristiche cliniche omogenee (Tab. 1).

\section{Risultati}

\section{Caratteristiche dei soggetti inclusi nello studio e caratteristiche dei trattamenti farmacologici}

Il numero dei trattamenti inclusi nello studio è stato di 604 , corrispondenti a 485 pazienti (più trattamenti possono essere riferiti a uno stesso paziente).

Sulla base dell'esperienza clinica dell'uso del sodio oxibato, i trattamenti inclusi nello studio sono stati divisi in due gruppi: 470 trattamenti per un periodo inferiore a 90 giorni e 134 trattamenti per un periodo superiore a 90 giorni. Per il primo gruppo, che ha ricevuto il sodio oxibato per un periodo inferiore a 90 giorni, l'outcome principale del trattamento è stato la riduzione della sindrome astinenziale, mentre per il secondo gruppo, che ha ricevuto il farmaco per un periodo superiore a 90 giorni, l'outcome principale è stata la riduzione del consumo alcolico. La media dei giorni di trattamento è stata di $40.49 \pm 24.58$ e di $236.89 \pm 278.10$ per il periodo corto e lungo rispettivamente, mentre la media della dose giornaliera di sodio oxibato (espressa in $\mathrm{mg} / \mathrm{kg} / \mathrm{die}$ ) è stata di $78.26 \pm 19.5 \mathrm{e}$ $76.61 \pm 24.3$ rispettivamente e (espressa in $\mathrm{ml} /$ die) è stata di $31.7 \pm 10.5$ e $29.67 \pm 11.3$ rispettivamente.

\section{Obiettivi primari di efficacia (efficacy) del far- maco}

\section{Effetti antiastinenziali}

Il sodio oxibato è stato in grado di ridurre la sindrome astinenziale, il consumo di alcol e il craving. In parti- colare il successo del trattamento è stato dell' $81 \%$ (Tab. 2).

Tab. 2 - Consumo di alcol, craving e percentuale di successo al trattamento di disintossicazione

\begin{tabular}{|c|c|c|}
\hline & $\begin{array}{l}\text { Teтро } 0 \\
(n=470)\end{array}$ & $\begin{array}{l}\text { Fine del } \\
\text { Trattamento } \\
(n=336)\end{array}$ \\
\hline Intake di alcol & $7.67+1.15$ & $0.63+0.90^{*}$ \\
\hline Alcol craving & $8.23+0.63$ & $2.51+1.23^{*}$ \\
\hline $\begin{array}{l}\text { Percentuale del successo } \\
\text { del trattamento }\end{array}$ & $=$ & $81 \%($ n. $=274)$ \\
\hline
\end{tabular}

$* p<0.05$ vs. tempo 0

Il successo del trattamento è definito quando la CIWA-Ar è $<7$

\section{Effetti di prevenzione delle ricadute}

Il sodio oxibato si è dimostrato capace di mantenere l'astinenza, di ridurre il craving e di normalizzare i parametri biochimici del consumo cronico di alcol (Tab. 3).

In particolare a un follow-up a 6 e 12 mesi la percentuale di soggetti astinenti è stata rispettivamente del 76 e del $78 \%$ (Tab. 4).

Tab. 3 - Consumo di alcol, craving e parametri biochimici del consumo di alcol: Follow-up a 6 e 12 mesi

\begin{tabular}{llll}
\hline & $\begin{array}{l}\text { Tempo 0 } \\
(n=134)\end{array}$ & $\begin{array}{l}\text { Follow-up } \\
6 \text { mesi } \\
(n=88)\end{array}$ & $\begin{array}{l}\text { Follow-up } \\
12 \text { mesi } \\
(n=18)\end{array}$ \\
\hline Intake di alcol & $\mathbf{8 . 7 2 + 1 . 2 2}$ & $0.95+1.92 *$ & $1+2.16^{*}$ \\
Alcol craving & $\mathbf{8 . 0 8 + 0 . 8 9}$ & $1.01+1.17 *$ & $1+1.13 *$ \\
MCV (fl) & $104.92+9.25$ & $\mathbf{8 8 . 6 9 + 5 . 1 6 ^ { * }}$ & $82.5+3.09 *$ \\
GGT (U/l) & $\mathbf{8 9 . 4 3 + 1 6 . 1 5}$ & $36.53+6.96^{*}$ & $36.94+6.88$ \\
AST (U/l) & $45.44+4.39$ & $28.35+4.95^{*}$ & $31.38+6.59 *$ \\
ALT (U/l) & $45.65+3.71$ & $31.76+7.96^{*}$ & $29.44+4.16^{*}$
\end{tabular}

Dati espressi come media \pm S.D.

$* p<0.05$ vs. tempo 0

Tab. 4 - Risposta al trattamento: Follow-up a 6 e 12 mesi

\begin{tabular}{llll}
\hline & $\begin{array}{l}\text { Tempo 0 } \\
(\mathrm{n}=134)\end{array}$ & $\begin{array}{l}\text { Follow-up } \\
6 \text { mesi } \\
(\mathrm{n}=88)\end{array}$ & $\begin{array}{l}\text { Follow-up } \\
12 \text { mesi } \\
(\mathrm{n}=18)\end{array}$ \\
\hline $\begin{array}{llll}\text { Astinente } \\
\text { Non astinente }\end{array}$ & $=$ & $76 \%(\mathrm{n}=67)$ & $78 \%(\mathrm{n}=14)$ \\
Ricaduto & $=$ & $16 \%(\mathrm{n}=14)$ & $11 \%(\mathrm{n}=2)$ \\
\hline
\end{tabular}

Astinente $=$ nessuna assunzione di alcol

Non astinente $=$ meno di $5 d r i n k$ in una occasione per gli uomini e meno di 4 drink in una occasione per le donne

Ricaduto nel bere $=5$ o più drink in una occasione per gli uomini e 4 o più drink in una occasione per le donne 


\section{Drop-out dal trattamento}

Il drop-out dal trattamento è stato rispettivamente del $28 \%(\mathrm{n}=134)$ e del $16 \%(\mathrm{n}=22)(P<0.05)$ nei soggetti in trattamento per un periodo inferiore e superiore ai 90 giorni.

Durante il periodo di trattamento a breve termine $(<90$ giorni) le cause di drop-out sono state per il $63 \%(\mathrm{n}=$ 85) legate ai pazienti e per il $37 \%(n=49)(P<0.05)$ al farmaco.

Le cause di drop-out legate al paziente sono state: decisione del paziente $(48 \%, n=41))$, nessuna compliance $(30 \%, \mathrm{n}=25)$, cambiamento del trattamento $(16 \%, \mathrm{n}=$ 14) e altro $(6 \%, \mathrm{n}=5)$, mentre quelle legate al farmaco sono state: effetti collaterali $(89 \%, \mathrm{n}=44)$ e intossicazioni $(10 \%, \mathrm{n}=5)$.

Durante il periodo di trattamento a lungo termine ( $>90$ giorni) le cause di drop-out sono state per il $90 \%(\mathrm{n}=$ 20) legate ai pazienti e per il $10 \%(n=2)(P<0.05)$ al farmaco.

Le cause di drop-out legate al paziente sono state: decisione del paziente $(70 \%, \mathrm{n}=14)$, nessuna compliance $(10 \%, \mathrm{n}=2)$ e cambiamento del trattamento $(20 \%, \mathrm{n}=$ $4)$, mentre quelle legate al farmaco sono state: effetti collaterali $(100 \%, \mathrm{n}=2)(P<0.05)$.

\section{Obiettivi secondari di sicurezza (safety) del farmaco}

\section{Affido farmacologico domiciliare}

L'82\% ( $\mathrm{n}=275)$ dei soggetti in trattamento per un periodo inferiore di 90 giorni ha ricevuto l'affido domiciliare del sodio oxibato.

A 6 e 12 mesi dall'inizio del trattamento il $100 \%(n=88$ e 18 rispettivamente) dei soggetti trattati ha ricevuto il farmaco in affido.

\section{Effetti collaterali}

Il $19 \%(n=64)$ dei soggetti trattati per un periodo inferiore di 90 giorni ha presentato effetti collaterali. A 6 e 12 mesi dall'inizio del trattamento non si sono registrati effetti collaterali. Gli effetti collaterali più frequenti sono stati le vertigini $(34 \%, \mathrm{n}=22)$, la nausea e il vomito $(8 \%, n=5)$, le convulsioni $(6 \%, n=4)$, altri effetti indesiderati $(33 \%, \mathrm{n}=21)$ e più effetti contemporanei $(19 \%, n=4)$.

\section{Reazioni avverse}

Le reazioni avverse al trattamento si sono verificate in una piccola percentuale di soggetti $(0.9 \%, \mathrm{n}=3)$ e sempre nelle prime fasi del trattamento ( $<90$ giorni) .

\section{Comportamenti di misuso}

Il $12 \%(n=79)$ dei soggetti in trattamento con sodio oxibato ha presentato dei comportamenti di misuso ${ }^{1}$; in particolare, la percentuale maggiore $(18 \%)$ di "misusers" si è registrata nelle prime fase del trattamento (<90 giorni) (Fig. 1).

Fig. 1 - Percentuale di "misusers"

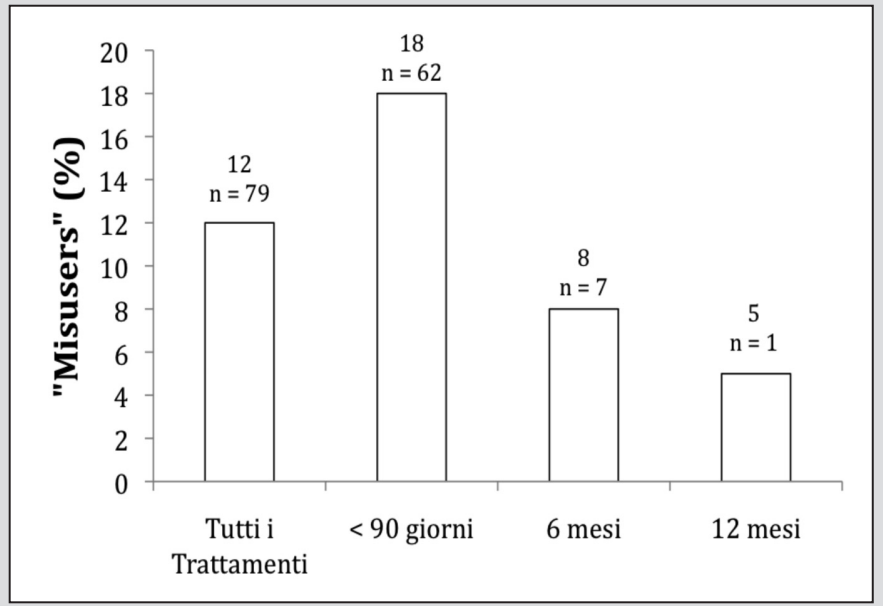

In Fig. 2 sono rappresentati i più frequenti comportamenti di misuso e "appetitivi" registrati nei soggetti in trattamento.

Essi sono stati: incremento del dosaggio (34\%), non osservazione del dosaggio (28\%) e co-assunzione del farmaco con altre sostanze (20\%); la co-assunzione di alcol si è presentata nel $14 \%$ e più comportamenti si sono manifestati nel $4 \%$.

Fig. 2 - Percentuale di comportamenti di misuso

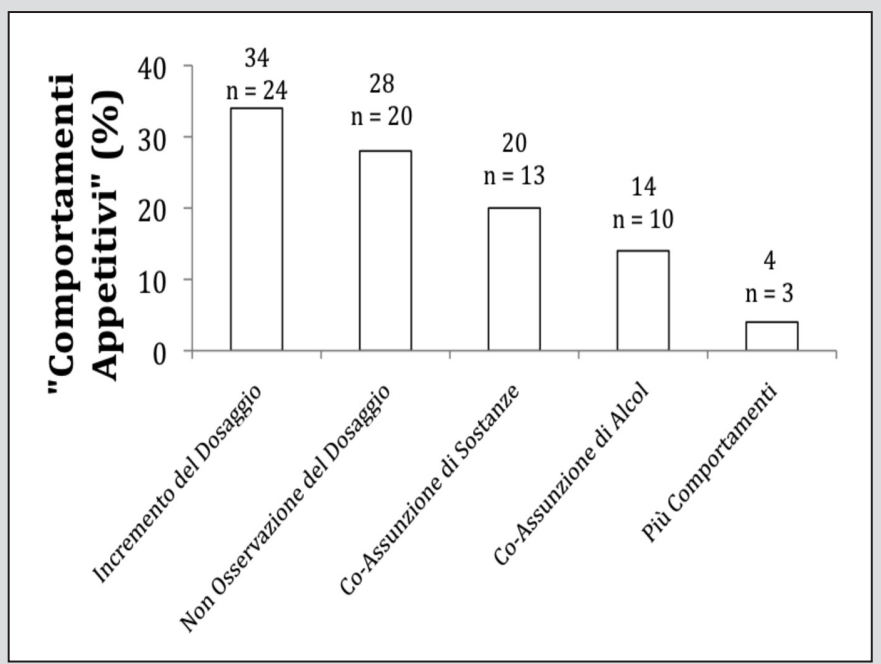

In Fig. 3 vengono rappresentate le principali caratteristiche dei "misusers", la maggior parte di loro sono poliassuntori $(26 \%)$, affetti da disturbi di personalità $(24 \%)$, con uso passato di sostanze $(21 \%)$, non astinen- 
ti o ricaduti (17\%) e affetti da disturbi psichiatrici $(12 \%)$.

Fig. 3 - Percentuale delle caratteristiche dei "misusers"

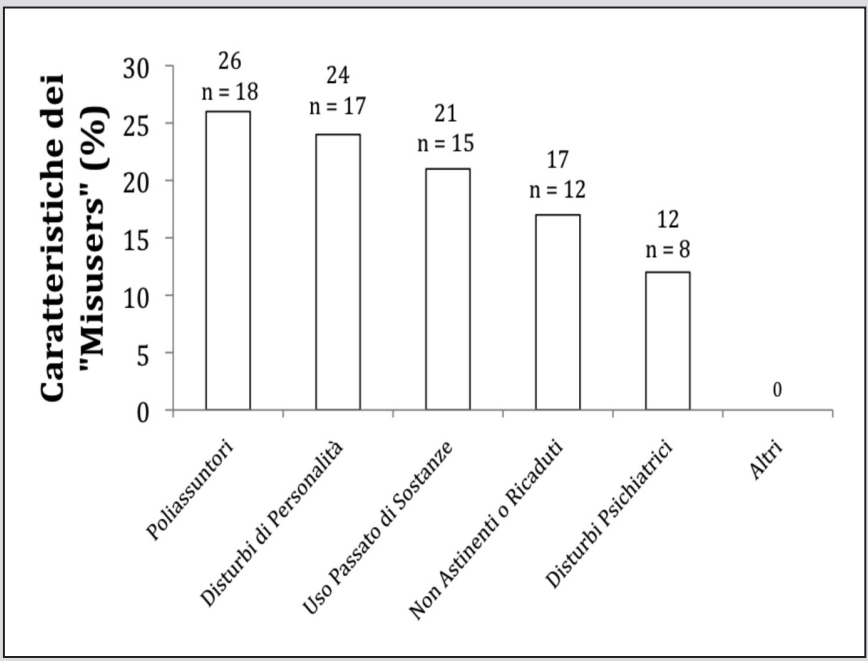

Per quanto riguarda le principali "azioni" ("contromisure") messe in atto dai medici per controllare i comportamenti di misuso esse sono state: incremento della supervisione nell'assunzione (29\%), sospensione dell'affido (21\%), maggiore frequenza dei controlli clinici (19\%), aumento del frazionamento giornaliero della dose $(19 \%)$ e solo in una percentuale minore (12\%) interruzione della prescrizione del farmaco (Fig. 4).

Fig. 4 - Percentuale delle "azioni" messe in atto dai medici per controllare il misuso

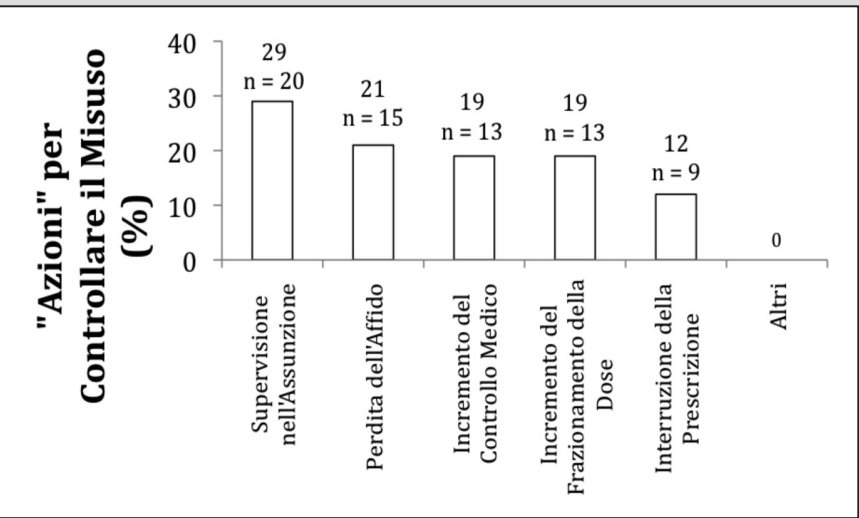

Overdose, Intossicazione e Astinenza

Nei casi presi in esame si è verificato un solo caso di overdose $(0.3 \%), 5$ casi di intossicazione $(1 \%)$ e 6 di astinenza (alla sospensione volontaria del farmaco) $(1 \%)$.

Tutti i casi si sono verificati durante il periodo breve del trattamento ( $<90$ giorni).

\section{Conclusioni}

Lo studio GUM rappresenta il primo e più importante studio retrospettivo sull'uso del sodio oxibato (Alcover $\left.^{\circledR}\right)$ nel trattamento dell'alcolismo condotto in Italia. Lo studio ha infatti preso in considerazione, nei 7 centri clinici selezionati, 604 trattamenti con sodio oxibato (corrispondenti a 485 pazienti trattati).

Il limite maggiore dello studio è che esso rappresenta una survey e non è stato realizzato sotto forma di studio clinico controllato.

Lo studio ha dimostrato che il sodio oxibato è efficace nel ridurre la sindrome astinenziale nell' $81 \%$ dei soggetti trattati e di mantenere l'astinenza a 6 e 12 mesi dal trattamento nel 76 ed il $78 \%$ dei pazienti.

Lo studio ha permesso di identificare le principali caratteristiche demografiche e cliniche dei soggetti in trattamento con sodio oxibato in Italia mettendo in evidenza come i soggetti valutati hanno una dipendenza alcolica probabilmente medio-grave e una età media elevata (circa 40 anni), sono (o sono stati) poliassuntori e sono affetti da una co-morbilità psichiatrica di Asse I e II del DSM-IV-TR.

Lo studio ha permesso di individuare il tempo e il dosaggio medio di trattamento con sodio oxibato dimostrando come la maggior parte dei trattamenti sono a breve termine (verosimilmente finalizzati a disintossicare $i$ pazienti) e come il farmaco viene usualmente utilizzato con un dosaggio medio compreso fra i valori consigliati (cioè fra i 50 e i $100 \mathrm{mg} / \mathrm{kg} / \mathrm{die}$ ).

I soggetti alcolisti in trattamento con sodio oxibato non hanno presentato una elevata percentuale di drop-out dal trattamento.

Questi si sono maggiormente verificati nelle prime fasi del trattamento ( $<90$ giorni) (28\% vs. $16 \%>90$ giorni) e le cause sono state per la maggior parte legate ai pazienti (che nella maggior parte dei casi hanno deciso volontariamente di interrompere il trattamento).

Fra le cause di drop-out legate al farmaco il maggior numero è stato dovuto all'insorgenza di effetti collaterali $(89 \%$ nel trattamento $<90$ giorni e $100 \%$ nel trattamento $>90$ giorni).

Il sodio oxibato viene percepito da parte dei medici come un farmaco sicuro tanto da essere concesso ai pazienti in affido domiciliare già durante le prime fasi del trattamento ( $<90$ giorni) nell' $82 \%$ dei casi e nel $100 \%$ dei casi nelle fasi più prolungate di trattamento (>90 giorni).

Lo studio ha confermato che il sodio oxibato è un farmaco maneggevole.

Gli effetti collaterali infatti sono stati contenuti (si sono presentati nel $19 \%$ dei trattamenti) e poche sono state le reazioni avverse $(0.9 \%)$.

Entrambi gli eventi si sono verificati nelle prime fasi del trattamento (<90 giorni). 
Lo studio ha confermato che gli effetti collaterali più frequenti sono le vertigini (34\%), la nausea e il vomito $(8 \%)$ e le convulsioni $(6 \%)$.

Lo studio ha dimostrato che il potenziale di misuso e di abuso del sodio oxibato è relativamente basso.

In maniera interessante lo studio ha messo in evidenza come il misuso, cioè l'utilizzo del farmaco fuori dalle indicazioni d'uso e prescrittive sia contenuto nei soggetti studiati $(12 \%$ in tutti i trattamenti, $18 \%<90$ giorni, $8 \%$ a 6 mesi e $5 \%$ a 12 mesi).

I comportamenti di misuso (anche definiti come comportamenti "appetitivi") più frequenti sono stati l'incremento del dosaggio prescritto $(34 \%)$, la non osservanza del dosaggio prescritto $(28 \%)$ e la co-assunzione del farmaco con sostanze $(20 \%)$ e alcol (14\%).

Anche in questo caso i comportamenti di misuso si sono maggiormente verificati durante le prime fasi del trattamento ( $<90$ giorni).

In maniera interessante lo studio ha permesso di identificare fra i fattori predittivi del misuso; la poliassunzione (come uso presente di sostanze nel $26 \%$ dei casi o uso passato nel $21 \%$ ), la presenza di un disturbo di personalità $(24 \%$ dei casi) o gravi disturbi psichiatrici $(12 \%$ dei casi).

I casi di abuso (intossicazione e overdose) del farmaco sono stati limitati (rispettivamente $1 \%$ e $0.3 \%$ ) e si sono verificati nelle prime fasi del trattamento $(<90$ giorni). Altrettanto limitati sono stati i casi di astinenza (1.3\%) che si sono verificati unicamente quando $\mathrm{i}$ pazienti hanno deciso di interrompere volontariamente e prematuramente il trattamento.

Lo studio ha dimostrato un buon profilo di sicurezza del sodio oxibato, soprattutto nei trattamenti di lunga durata e quando il farmaco è stato utilizzato a dosaggi compresi fra i 50 e i $100 \mathrm{mg} / \mathrm{kg} /$ die.

I dati sul misuso e sull'abuso del sodio oxibato del presente studio sono coerenti con quelli di alcune revisioni sull'utilizzo del sodio oxibato nel trattamento della dipendenza da alcol che hanno dimostrato come il craving per il sodio oxibato è limitato (?10-15\% dei soggetti trattati) (Caputo e coll., 2009a,b) e come il rischio di abuso del sodio oxibato sia generalmente basso quando viene somministrato a pazienti con dipendenza da alcol secondo il dosaggio approvato (compreso fra i 50 e i 100 $\mathrm{mg} / \mathrm{kg} / \mathrm{die}$ ) (Keating, 2014), sotto supervisione di un familiare designato e con sorveglianza medica stretta e continuativa. D'altra parte lo studio GUM dimostra come pazienti con dipendenza da alcol e disturbo di personalità borderline o in remissione da dipendenza da eroina o cocaina, non siano, in assoluto, i candidati adatti per il trattamento con sodio oxibato perché mostrano un rischio aumentato di abuso, sebbene le evidenze cliniche dimostrino che il rischio di abuso rimane relativamente basso se precocemente riconosciuto e prevenuto (Skala e coll., 2014); per tali ragioni, il sodio oxi- bato deve essere prescritto e monitorato dai medici della medicina delle dipendenze.

Riassumendo, possiamo affermare, come ribadito in uno studio relativo alle nuove strategie di trattamento della dipendenza da alcol con il sodio oxibato nella pratica clinica a cura di Caputo e colleghi (2015), che il rischio di abuso del sodio oxibato è basso (?10\% dei soggetti trattati) quando viene somministrato al dosaggio raccomandato, sotto supervisione di un membro della famiglia designato e con sorveglianza medica stretta e continuativa; in ogni caso il rischio di sviluppare abuso del sodio oxibato può essere presente in pazienti con poliabuso di sostanze e con co-morbilità psichiatrica (in particolare disturbo borderline di personalità).

In conclusione, lo studio GUM conferma l'efficacia del sodio oxibato (Alcover ${ }^{\circledR}$ ) come farmaco antiastinenziale, per il mantenimento dell'astinenza e anti-craving, e dimostra come le potenzialità di misuso e abuso del farmaco possono essere limitate nella pratica clinica.

I dati sopra esposti e $i$ commenti riportati, che hanno costituito la relazione presentata nella Sessione parallela D "L'efficacia e la sicurezza del sodio oxibato nella pratica clinica all'interno del Convegno Tematico Nazionale DIVERSIONE E MISUSO Firenze 15-16 ottobre 2015 presso Palaffari-FirenzeFiera, sono stati tratti dal report finale dei risultati dello Studio GUM (The GHB Use and Misuse GUM-Study) (Studio Retrospettivo dell'uso del Sodio Oxibato-Alcover ${ }^{\circledR}$ nel Nord Italia).

Lo studio è stato eseguito con il supporto del Laboratorio Farmaceutico C.T.

\section{Note}

1. Per comportamenti "appetitivi" si intende l'utilizzo del farmaco per scopi diversi da quelli terapeutici.

Fra essi i più importanti sono l'incremento e la non osservanza del dosaggio prescritto e la co-assunzione del farmaco con altre sostanze e/o l'alcol.

\section{Bibliografia}

Addolorato G., Castelli E., Stefanini G.F., Casella G., Caputo F., Marsigli L., Bernardi M., Gasparini G. (1996), "An open multicentric study evaluating gamma-hydroxybutyric acid sodium salt in the mediumterm treatment of 179 alcohol dependent subjects", Alcohol and Alcoholism, 31: 341-345.

Addolorato G., Cibin M., Caputo F., Capristo E., Gessa G.L., Stefanini G.F., Gasbarrini G. (1998), " $\gamma$-Hydroxybutyric acid in the treatment of alcoholism: dosage fractioning utility in non-responder alcoholic patients", Drug and Alcohol Dependence, 53: 7-10.

Addolorato G., Balducci G., Capristo E., Attilia M.L., Taggi F., Gasbarrini G., Ceccanti M. (1999a), "Gamma-hydroxybutyric acid (GHB) in the treatment of alcohol withdrawal syndrome: a randomized comparative study versus benzodiazepine", Alcoholism: Clinical and Experimental Research, 23: 1596-1604. 
Addolorato G., Cibin N., Caputo F., Capristo E., Gessa G.L., Stefanini G.F., Gasbarrini G. (1999b), "Gamma-hydroxybutyric acid in the treatment of alcoholism: dosage fractioning utility in non-responder alcoholic patients", Drug and Alcohol Dependence, 53: 7-10.

Addolorato G., Caputo F., Capristo E., Stefanini G.F., Gasbarrini G. (2000), "Gamma-hydroxybutyric acid efficacy, potential abuse, and dependence in the treatment of alcohol addiction", Alcoholism, 53: 7 10.

Caputo F., Vignoli T., Maremmani I., Bernardi M., Zoli G. (2009a), "Gamma hydroxybutiric acid (GHB) for the treatment of alcohol dependence: A review", International Journal of Environmental Research and Public Health, 6: 1917-1929.

Caputo F., Francini S., Stoppo M., Lorenzini F., Vignoli T., Del Re A., Comaschi C., Leggio L., Addolorato G., Zoli G., Bernardi M. (2009b), "Incidence of craving for and abuse of gamma-hydroxybutyric acid (GHB) in different populations of treated alcoholics: an open comparative study", Journal of Psychopharmacology, 23: 883-890.

Caputo F., Francini S., Brambilla R., Vigna-Taglianti F., Stoppo M., Del Re A., Leggio L., Addolorato G., Zoli G., Bernardi M. (2011), "Sodium oxybate in maintaining alcohol abstinence in alcoholic patients with and without psychiatric comorbidity", European Neuropsychopharmacology, 21: 450-456.

Caputo F., Skala K., Walter H., Ceccanti M., Djurkowski M., Filipecka E., Florkowski A., Gerra G., Holzbach R., Horodnicki J., Platz W., Spazzapan B., Zblowska H., Bernardi M., Cacciaglia R., Vivet P., Lesch O.M., Addolorato G. (2013), "Sodium oxibate in the prevention of alcohol relapses in alcohol dependent patients (GATE 2 Study)", Alcohol and Alcoholism, 48: S1-i33.

Caputo F., Mirijello A., Cibin M., Mosti A., Ceccanti M., Domenicali M., Bernardi M., Maremmani I., Addolorato G., For the "Twentieth Anniversary of the Use of SMO in Italy" Group (2015), "Novel strategies to treat alcohol dependence with sodium oxybate according to clinical practice", European Review for Medical and Pharmacological Sciences, 19: 1315-1320.

Keating G.M. (2014), "Sodium oxybate: A review of its use in alcohol withdrawal syndrome and in the maintenance of abstinence in alcohol dependence", Clinical Drug Investigation, 34: 63-80.

Nava F., Premi S., Manzato E., Lucchini A. (2006), "Comparing treatment of alcoholism on craving and biochemical measures of alcohol consumption", Journal of Psychoactive Drugs, 38: 211-217.

Nava F., Premi S., Manzato E., Campagnola W., Lucchini A., Gessa G.L. (2007), "Gamma-hydroxybutyrate reduces both withdrawal syndrome and hypercortisolism in severe abstinent alcoholics: an open study vs. diazepam", American Journal of Drug and Alcohol Abuse, 33: 379-392.

Nava F., Vendramin A., Manzato E., Cibin M., Lucchini A. (2010), "New frontiers in alcoholism and addiction treatment", Recent Patents on CNS Drug Discovery, 5: 81-94.

Skala K., Caputo F., Mirijello A., Vassallo G. Antonelli M., Ferrulli A., Walter H., Lesch O.M., Addolorato G. (2014), "Sodium oxybate in the treatment of alcohol dependance: from the alcohol withdrawal syndrome to the alcohol relapse prevention", Export Opinion on Pharmacotherapy, 15: 245-257.

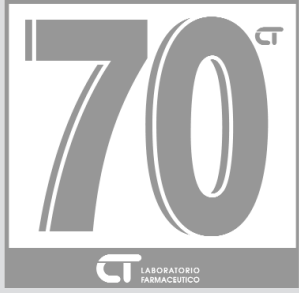

Si ringrazia il Laboratorio Farmaceutico C.T. per il supporto redazionale alla realizzazione della newsletter. 